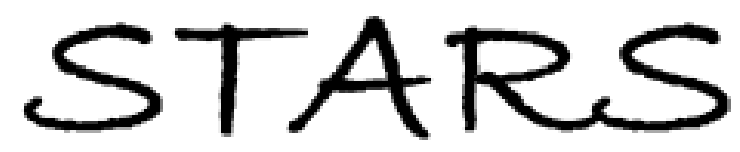

University of Central Florida

STARS

$1-1-2014$

\title{
Electro-optic response of polymer-stabilized blue phase liquid crystals
}

\author{
Daming $\mathrm{Xu}$ \\ University of Central Florida \\ Jiang Yan \\ University of Central Florida \\ Jiamin Yuan \\ University of Central Florida \\ Fenglin Peng \\ University of Central Florida \\ Yuan Chen \\ University of Central Florida
}

Find similar works at: https://stars.library.ucf.edu/facultybib2010

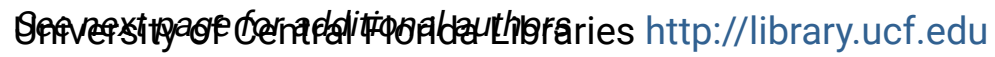

This Article is brought to you for free and open access by the Faculty Bibliography at STARS. It has been accepted for inclusion in Faculty Bibliography 2010 s by an authorized administrator of STARS. For more information, please contactSTARS@ucf.edu.

\section{Recommended Citation}

Xu, Daming; Yan, Jiang; Yuan, Jiamin; Peng, Fenglin; Chen, Yuan; and Wu, Shin-Tson, "Electro-optic response of polymer-stabilized blue phase liquid crystals" (2014). Faculty Bibliography 2010s. 6313. https://stars.library.ucf.edu/facultybib2010/6313

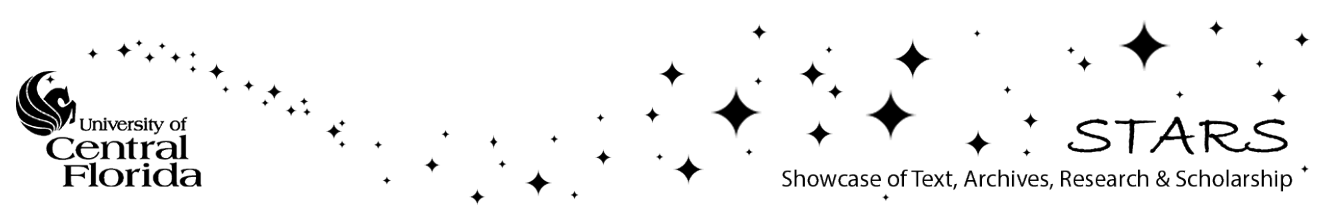




\section{Authors}

Daming Xu, Jiang Yan, Jiamin Yuan, Fenglin Peng, Yuan Chen, and Shin-Tson Wu 


\section{Electro-optic response of polymer-stabilized blue phase liquid crystals}

Cite as: Appl. Phys. Lett. 105, 011119 (2014); https://doi.org/10.1063/1.4890031

Submitted: 19 June 2014 . Accepted: 01 July 2014 . Published Online: 09 July 2014

Daming Xu, Jing Yan, Jiamin Yuan, Fenglin Peng, Yuan Chen, and Shin-Tson Wu

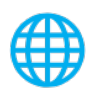

\section{ARTICLES YOU MAY BE INTERESTED IN}

Electro-optics of polymer-stabilized blue phase liquid crystal displays

Applied Physics Letters 94, 101104 (2009); https://doi.org/10.1063/1.3097355

Extended Kerr effect of polymer-stabilized blue-phase liquid crystals

Applied Physics Letters 96, 071105 (2010); https://doi.org/10.1063/1.3318288

A large Kerr constant polymer-stabilized blue phase liquid crystal

Applied Physics Letters 98, 081109 (2011); https://doi.org/10.1063/1.3559614

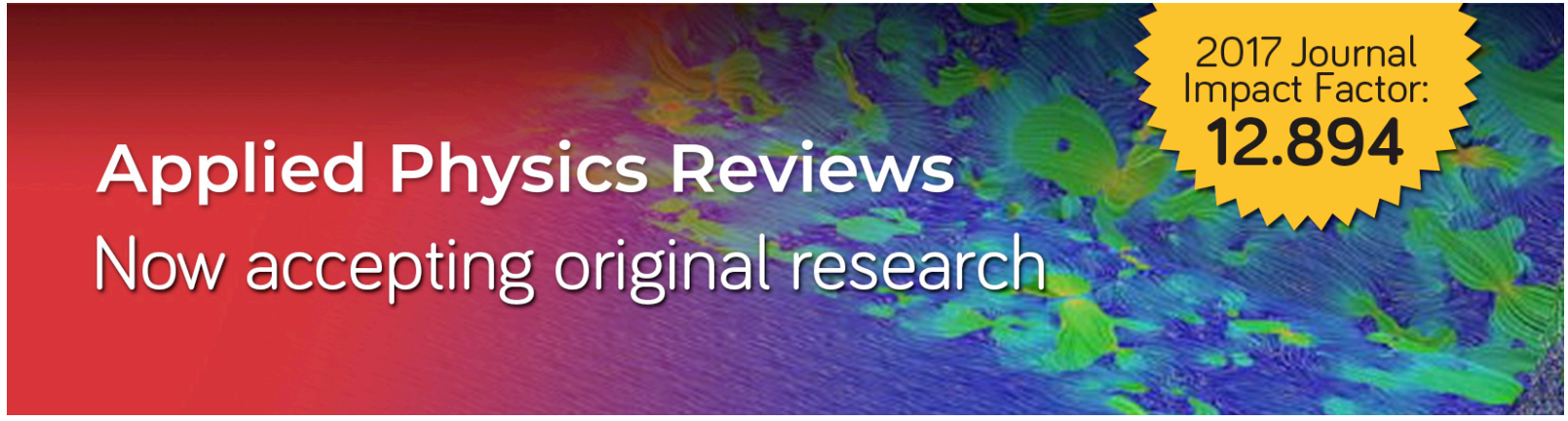




\title{
Electro-optic response of polymer-stabilized blue phase liquid crystals
}

\author{
Daming Xu, ${ }^{1}$ Jing Yan, ${ }^{1,2}$ Jiamin Yuan, ${ }^{1}$ Fenglin Peng, ${ }^{1}$ Yuan Chen, ${ }^{1}$ and Shin-Tson $\mathrm{Wu}{ }^{1, a)}$ \\ ${ }^{1}$ CREOL, The College of Optics and Photonics, University of Central Florida, Orlando, Florida 32816, USA \\ ${ }^{2}$ Display Center, School of Electronic Science and Engineering, Southeast University, Nanjing, China
}

(Received 19 June 2014; accepted 1 July 2014; published online 9 July 2014)

\begin{abstract}
The dynamic response of a polymer-stabilized blue phase liquid crystal (BPLC) is comprised of two distinct processes: Kerr effect-induced local reorientation and electrostriction-induced lattice distortion. A double exponential rise/decay model is proposed to analyze the underlying physical mechanisms. If the electric field is below a critical field $\left(E_{c}\right)$, Kerr effect dominates and the response time is fast. However, when $E>E_{c}$ electrostriction effect manifests, leading to an increased response time and a noticeable hysteresis. A higher polymer concentration helps suppress electrostriction, but the tradeoff is increased operation voltage. These results provide useful guidelines for future BPLC material and device optimizations. (C) 2014 AIP Publishing LLC.

[http://dx.doi.org/10.1063/1.4890031]
\end{abstract}

Polymer-stabilized blue phase liquid crystal (PSBPLC) ${ }^{1}$ is a promising candidate for next-generation display $^{2,3}$ and photonic applications. ${ }^{4,5}$ Compared to nematic liquid crystals, ${ }^{6,7}$ BPLC exhibits following attractive features: (1) Self-assembled three-dimensional lattice structure so that no surface alignment layer is required; (2) nanoscale double-twisted cylinder diameter and short coherence length, which result in submillisecond response time, ${ }^{8,9}$ and (3) optically isotropic voltage-off state which leads to polarization independent phase modulation. ${ }^{4}$ Among these features, fast response time is the most desirable one as it not only reduces motion blurs but also enables color sequential displays. ${ }^{10,11}$ The elimination of spatial color filters triples optical efficiency and resolution density. However, it was recently found that the response time of a PS-BPLC could vary from microseconds to several milliseconds, ${ }^{9,12}$ depending on the LC viscosity, pitch length, polymer network, and electric field strength. There is an urgent need to understand the dynamic response behaviors of a BPLC under different electric fields in order to improve the device performance.

As the electric field $(E)$ increases, three possible structure changes in a PS-BPLC could occur: local director reorientation governed by Kerr effect, ${ }^{13}$ lattice distortion induced by electrostriction effect, ${ }^{14,15}$ and finally phase transition to a lower symmetry phase. ${ }^{16}$ From macroscopic viewpoint, BPLC is optically isotropic at the voltage-off state. Upon application of an electric field, the isotropic medium becomes anisotropic, and the induced birefringence can be described by extended Kerr effect. Microscopically, the change in refractive index mainly originates from the local reorientation of nanoscale nematic domains confined in the double-twisted cylinders. Because of the short helical pitch of BPLC, the response time of this effect is usually in the submillisecond range, ${ }^{9}$ which is much faster than the Freedericksz transition in a nematic LC device. As $E$ increases, the polymer network could be deformed and the three-dimensional lattice of BPLC could be distorted accordingly, leading to electrostriction effect. ${ }^{14,15}$ Since the distortion of BPLC lattice usually involves as many as $10^{7} \mathrm{LC}$ molecules, its characteristic response time is usually

${ }^{a)}$ Electronic mail: swu@ucf.edu much longer than that of the Kerr effect; in the order of several milliseconds or longer. ${ }^{16}$ As $E$ continues to increase, diverse changes such as pitch dilation and phase transition to chiral nematic phase could occur. The helical structure of BPLC lattices are unwound, resulting in irreversible structural change. ${ }^{17,18}$ This process would take a much longer time than electrostriction. Therefore, electrostriction and phase transition are two major sources causing hysteresis and slow response time. To avoid these problems, ideally, a BPLC device should be operated below its critical field so that these two annoying effects would be suppressed. ${ }^{19}$

In this Letter, we investigate the dynamic response behavior of some BPLC composites under different electric fields. We find that electrostriction effect is the root cause for the observed slow response time and hysteresis in the high field region. PS-BPLCs with different monomer concentrations are also studied. Our experimental results show that a higher polymer concentration helps suppress electrostriction and achieve faster response time, but the tradeoff is increased operation voltage. Moreover, there is a critical field $\left(E_{c}\right)$ determining the onset of electrostriction and hysteresis.

In experiments, we employed a large $\Delta \varepsilon$ nematic LC host JC-BP06N (JNC, Japan) whose physical properties are listed as follows: $\Delta n=0.156$ at $\lambda=633 \mathrm{~nm}, \Delta \varepsilon=473.1$ at $100 \mathrm{~Hz}$ and $23^{\circ} \mathrm{C}$, and clearing temperature $T_{c}=73.8^{\circ} \mathrm{C}$. The BPLC host was then mixed with chiral dopant R5011 ( $\mathrm{HCCH}$, China) and two photocurable monomers: TMPTA $(1,1,1-$ Trimethylolpropane Triacrylate, Sigma Aldrich) and RM-257 (Merck). ${ }^{20}$ To investigate how the polymer network affects the electro-optic response of a PS-BPLC, we varied the monomer concentration while keeping the respective weight ratio between LC host and chiral dopant unchanged (96.8\%: $3.2 \%$ ), as listed in Table I. The weight ratio between RM-257 and TMPTA was kept at $\sim 1.5: 1$. To rule out the influence of nonuniform electric field in a commonly employed in-plane switching (IPS) cell, ${ }^{21}$ we injected the BPLC precursor into a vertical field switching (VFS) cell. ${ }^{12,22}$ The VFS cell was made of two planar ITO (indium tin oxide) glass substrates without surface alignment layer, and the electric field is in the longitudinal direction. Table I lists the compositions of three 
TABLE I. Material recipes and cell gap of three samples.

\begin{tabular}{llll}
\hline \hline & Sample 1 & Sample 2 & Sample 3 \\
\hline JC-BP06N & $88.33 \%$ & $85.21 \%$ & $85.54 \%$ \\
R5011 & $2.95 \%$ & $2.87 \%$ & $2.85 \%$ \\
RM257 & $5.26 \%$ & $6.75 \%$ & $7.01 \%$ \\
TMPTA & $3.46 \%$ & $4.17 \%$ & $4.60 \%$ \\
Cell gap & $7.84 \mu \mathrm{m}$ & $7.83 \mu \mathrm{m}$ & $7.77 \mu \mathrm{m}$ \\
\hline \hline
\end{tabular}

samples studied and their respective cell gaps. Next, we placed the VFS cell on a Linkam temperature controllable stage and let it cool to a temperature near the chiral nematic and blue phase transition temperature, and then cured it at BP-I with a UV light $\left(\lambda \sim 365 \mathrm{~nm}\right.$, intensity $\left.2 \mathrm{~mW} / \mathrm{cm}^{2}\right)$ for $30 \mathrm{~min}$. After UV exposure, the nanostructured BPLC composite was self-assembled.

Figure 1 depicts the experimental setup, in which the transmission axes of polarizer and analyzer are at $45^{\circ}$ and $-45^{\circ}$ azimuthal angles with respect to the incident plane. For intensity modulation with a VFS cell, the incident light should be at an oblique angle. Therefore, we immersed the VFS cell in a glass container filled with glycerol $(n=1.47)$, whose refractive index matches with that of BPLC. This enables the incident light to pass through the BPLC at a large angle in order to obtain more phase retardation. In our experiment, $\theta$ was set at $45^{\circ}$. A $100 \mathrm{~Hz}$ square-wave AC signal was applied to drive the VFS cell.

To measure rise time, we applied a voltage to the BPLC sample and recorded the transmittance change by a digital oscilloscope. Similarly, to measure decay time we removed the on-state voltage of the BPLC sample instantaneously and recorded the transient transmittance change. In our experiments, the analyzer was rotated by a small angle $\left(\sim 2^{\circ}\right)$ to compensate the polarization rotation effect originated from the BPLC layer. As a result, the measured contrast ratio is over 2000:1.23

The measured change in transmittance $T$ is related to the phase retardation $\delta$ as

$$
T=\sin ^{2}(\delta / 2)
$$

To rule out the cell gap effect and only focus on the intrinsic electro-optic properties of PS-BPLC, here, we converted the phase retardation to induced birefringence $\left(\Delta \mathrm{n}=n_{e}-n_{o}\right)$ using following equations: ${ }^{12}$

$$
\delta=\frac{2 \pi}{\lambda} n_{o} d\left[\sqrt{1-\frac{n_{g}^{2} \sin ^{2} \theta}{n_{e}^{2}}}-\sqrt{1-\frac{n_{g}^{2} \sin ^{2} \theta}{n_{o}^{2}}}\right],
$$

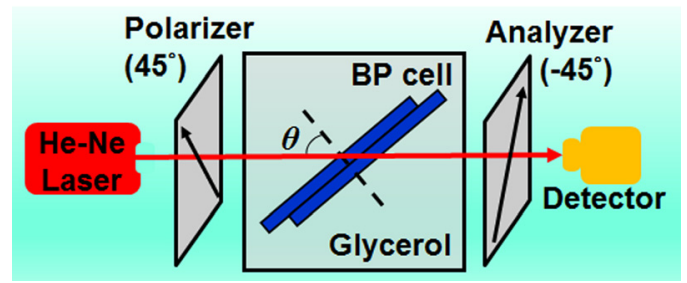

FIG. 1. Experimental setup for characterizing the dynamic response of a VFS cell.

$$
\begin{gathered}
n_{o}=n_{i}-\Delta n / 3, \\
n_{e}=n_{i}+2 \Delta n / 3
\end{gathered}
$$

where $\lambda$ is the laser wavelength, $n_{g}$ is the refractive index of glycerol, $\theta$ is the incident angle in the glycerol, $n_{i}$ is the refractive index in the voltage-off state, and $n_{e}$ and $n_{o}$ represent the extraordinary and ordinary refractive indices, respectively.

In Figs. 2(a) and 2(b), the black solid lines show the measured transient $\Delta n$ change during rise and decay processes using sample 2 as an example when an electric field $E=3 \mathrm{~V} / \mu \mathrm{m}$ was applied and then removed. It is clearly observed that both rise and decay curves involve two processes: a fast one followed by a slow one. This phenomenon originates from different response time scales between Kerr effect and electrostriction effect.

To further understand the electric field effects in the transient $\Delta n$ change process, we used exponential equations to fit the measured data. Although the Kerr effect dominates at low electric field as discussed above, a single exponential equation does not fit the experimental results too well, as shown by the blue dashed lines in Figs. 2(a) and 2(b) $\left(\mathrm{R}^{2}=91.6 \%\right.$ and $92.6 \%$ for rise and decay, respectively). Therefore, we need to include the electrostriction effect, although its contribution is very small. The following double exponential growth equation is better to describe the dynamic processes involving these two effects

$$
\Delta n(t)=A \times\left(1-e^{-t / t_{r 1}}\right)+B \times\left(1-e^{-t / t_{r 2}}\right),
$$
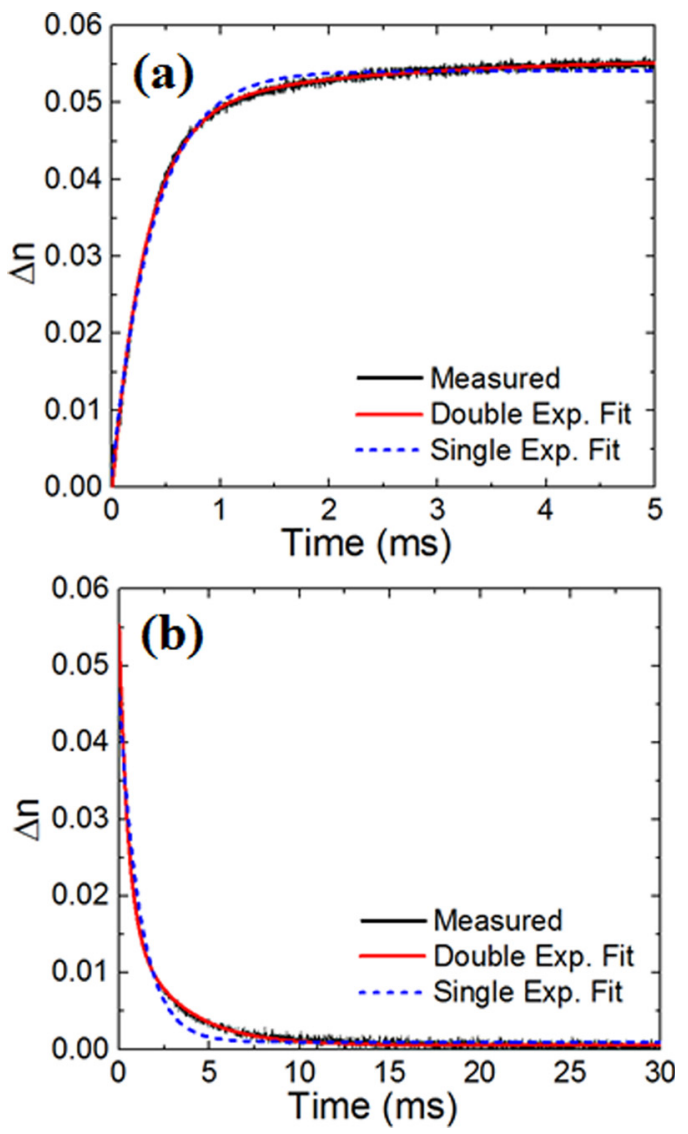

FIG. 2. Transient rise (a) and decay (b) processes of sample 2 under an applied electric field of $3 \mathrm{~V} / \mu \mathrm{m}$. The red solid and blue dashed lines are fitting results using double and single exponential equations, respectively. 
where $t_{r 1}$ and $t_{r 2}$ are the rise time constants, while $A$ and $B$ represent the induced birefringence from Kerr effect and electrostriction effect, respectively. For the decay process, we fitted the $\Delta n$ decay curve with following double exponential relaxation equation: ${ }^{24}$

$$
\Delta n(t)=C \times e^{-t / t_{d 1}}+D \times e^{-t / t_{d 2}},
$$

where $t_{d 1}$ and $t_{d 2}$ are the decay time constants, whereas $C$ and $D$ are the respective birefringence induced by the Kerr effect and electrostriction. The fitting curves (red solid lines) in Fig. 2 show that Eqs. (5) and (6) well characterize the rise and decay processes for $\Delta n\left(\mathrm{R}^{2}>99.7 \%\right.$ for both rise and decay fittings).

In experiments, we measured the rise time behaviors of all three samples under different electric fields ascending from $1 \mathrm{~V} / \mu \mathrm{m}$ to $11 \mathrm{~V} / \mu \mathrm{m}$, and fitted the experimental data with Eq. (5). The values of $t_{r 1}$ and $t_{r 2}$ under different electric fields are plotted in Figs. 3(a) and 3(b). All the values of $t_{r 1}$ shown in Fig. 3(a) are less than $1 \mathrm{~ms}$, which is well correlated with the response time of Kerr effect. In addition, Fig. 3(a) shows a clear trend: $t_{r 1}$ decreases as $E$ increases. This is because the local reorientation of nematic LCs inside the double-twisted cylinders experiences a stronger torque and reacts faster when the electric field gets stronger. ${ }^{8}$ More interestingly, in spite of the difference in monomer concentration, the $t_{r 1}$ of these three samples overlaps with each other. Therefore, $t_{r 1}$ is mainly determined by the LC host and chiral dopant; it is insensitive to the monomer weight ratio. This is because all the monomers are polymerized to form polymer network during UV curing and the properties (rotational viscosity $\gamma_{1}$, average elastic constant $k$, pitch length $P_{0}$, etc.) of the double-twisted BPLC structures are determined by the LC host and chiral dopant. Therefore, for those samples with same ratio between LC host and chiral dopant they form nearly the same BPLC lattice and the local LC reorientations within BPLC lattice have almost identical response time under an electric field. For electrostriction effect, similar dependency on electric field is shown in Fig. 3(b): as $E$ increases, $t_{r 2}$ decreases. This is because a strong electric field speeds up the electrostriction effect. The magnitude of $t_{r 2}$ is several milliseconds, which is much longer than $t_{r 1}$. Moreover, the value of $t_{r 2}$ depends on the monomer concentration; or more precisely the rigidness of polymer network. In a PS-BPLC with higher monomer concentration, the polymer network is more robust to resist lattice from distortion, which helps suppress the electrostriction effect. Therefore, it takes less time for the electric field to finish the BPLC lattice stretching process, as Fig. 3(b) depicts.

To compare the respective contributions of Kerr effect and electrostriction to the overall $\Delta n$, we plotted $A /(A+B)$ in Fig. 3(c). It is interesting to note that for each sample there is a critical electric field $E_{c}$, after which $A /(A+B)$ decreases dramatically. Let us take sample $1(8.72 \%$ monomer concentration) as an example. From Fig. 3(c), $A /(A+B)$ lies between 0.8 and 0.9 and it remains constant when $E<E_{c}=4 \mathrm{~V} / \mu \mathrm{m}$. This implies that in the low field region the LC local reorientation caused by Kerr effect plays a dominant role, while electrostriction effect is relatively weak. However, as $E>E_{c}$ the $A /(A+B)$ ratio starts to decrease, indicating that polymer
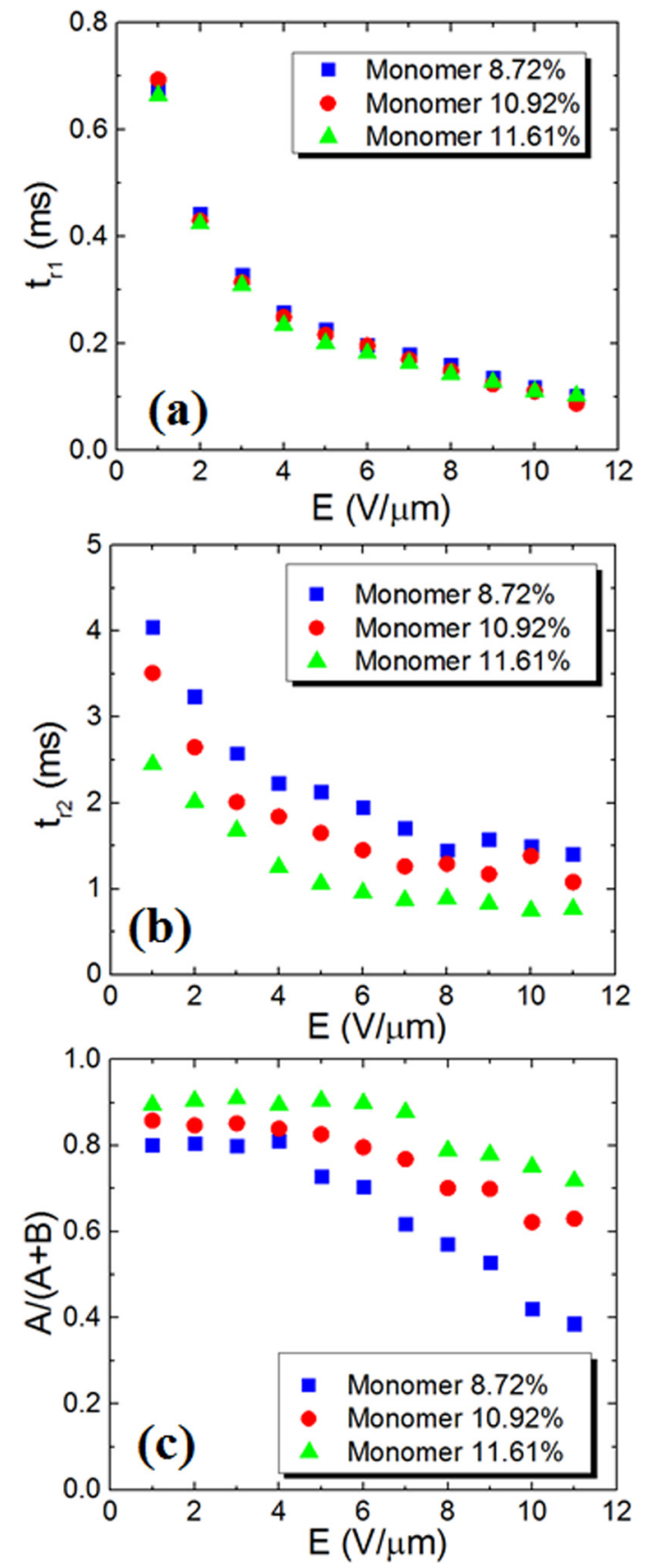

FIG. 3. (a) Measured rise time constant $t_{r 1}$ of Kerr effect, (b) rise time constant $t_{r 2}$ of electrostriction effect, and (c) respective contribution of Kerr effect when different electric fields are applied to samples with different monomer concentrations.

network and BPLC lattice are gradually deformed by the strong electric field. As a result, the electrostriction effect starts to make more contribution to the overall induced birefringence. For the sample with higher polymer concentration, whose polymer network is more rigid so that the electrostriction effect manifests at a higher critical field.

The critical field is not only the transition point of electrostriction effect but also an onset for hysteresis. We measured the hysteresis loops of each sample under different electric fields by ascending the voltage to a certain level and then descended it to zero. Due to the limitation of space, here, we only show the hysteresis curves of sample 1, while the other two samples also exhibit the same trend. The 


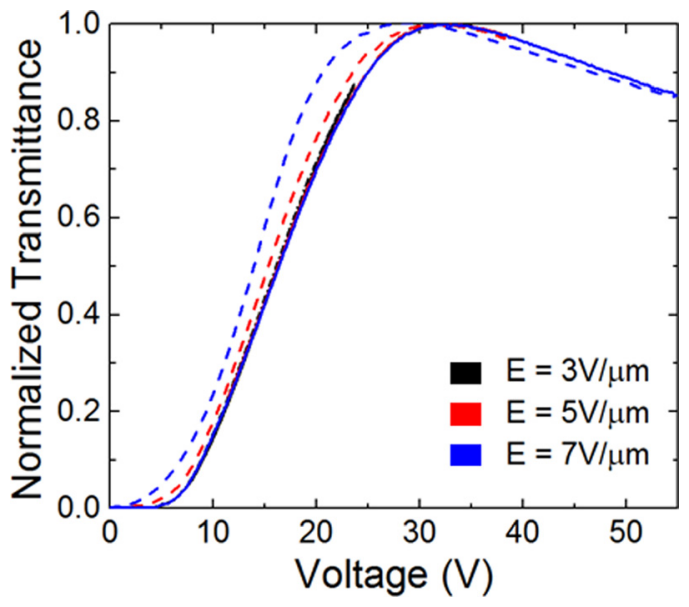

FIG. 4. Hysteresis of sample-1 under different electric fields: Solid lines represent forward driving and dashed lines backward driving.

measured hysteresis loops of sample 1 under different electric fields of 3, 5, and $7 \mathrm{~V} / \mu \mathrm{m}$ are plotted in Fig. 4, in which solid and dashed lines represent the VT (voltage-transmittance) curves under forward and backward driving, respectively. As clearly shown in Fig. 4, when $E<E_{c}(4 \mathrm{~V} / \mu \mathrm{m})$, the forward and backward VT curves overlap well with each other and the hysteresis is unnoticeable. This means there is no deformation in polymer network and the electrostriction effect is negligible. However, as we increase the electric field to $5 \mathrm{~V} /$ $\mu \mathrm{m}$ (which is higher than $E_{c}$ ), a small hysteresis is observed, which indicates the polymer network starts to be deformed and electrostriction effect becomes significant. At $E=7 \mathrm{~V} /$ $\mu \mathrm{m}$, the polymer network is deformed further and the lattice distortion is more severe, thus, the hysteresis becomes larger. One approach to suppress electrostriction effect and hysteresis is to increase polymer concentration, ${ }^{25,26}$ however, the tradeoff is increased operation voltage.

We also measured the transient decay process of all three samples under different electric fields, and fitted the experimental data with Eq. (6). Figures 5(a) and 5(b) depict the extracted decay time constants $t_{d 1}$ and $t_{d 2}$ under different electric fields. Similar to the rise time constant $t_{r 1}$ of Kerr effect, the decay time constant $t_{d 1}$ is also insensitive to the monomer concentration; it only depends on the weight ratio between LC host and chiral dopant. According to Gerber's model, ${ }^{27}$ the characteristic response time of Kerr effect is governed by rotational viscosity $\gamma_{1}$, average elastic constant $k$, and pitch length $P_{0}$ as follows:

$$
t_{d 1} \sim \tau_{0}=\frac{\gamma_{1} P_{0}^{2}}{4 \pi^{2} k} .
$$

Therefore, $t_{d 1}$ remains almost the same for PS-BPLC composites under same electric field as long as the ratio between LC host and chiral dopant does not change. However, in contrast to $t_{r 1}, t_{d 1}$ increases as the electric field gets stronger. This is because the local BPLC directors within double-twisted cylinders are unwound more severely under a stronger electric field. Therefore, upon removal of electric field it takes longer time for those BPLC directors to relax back to their original double-twisted structures, resulting in longer decay time. The same trend applies to $t_{d 2}$ as well, and this explains why the
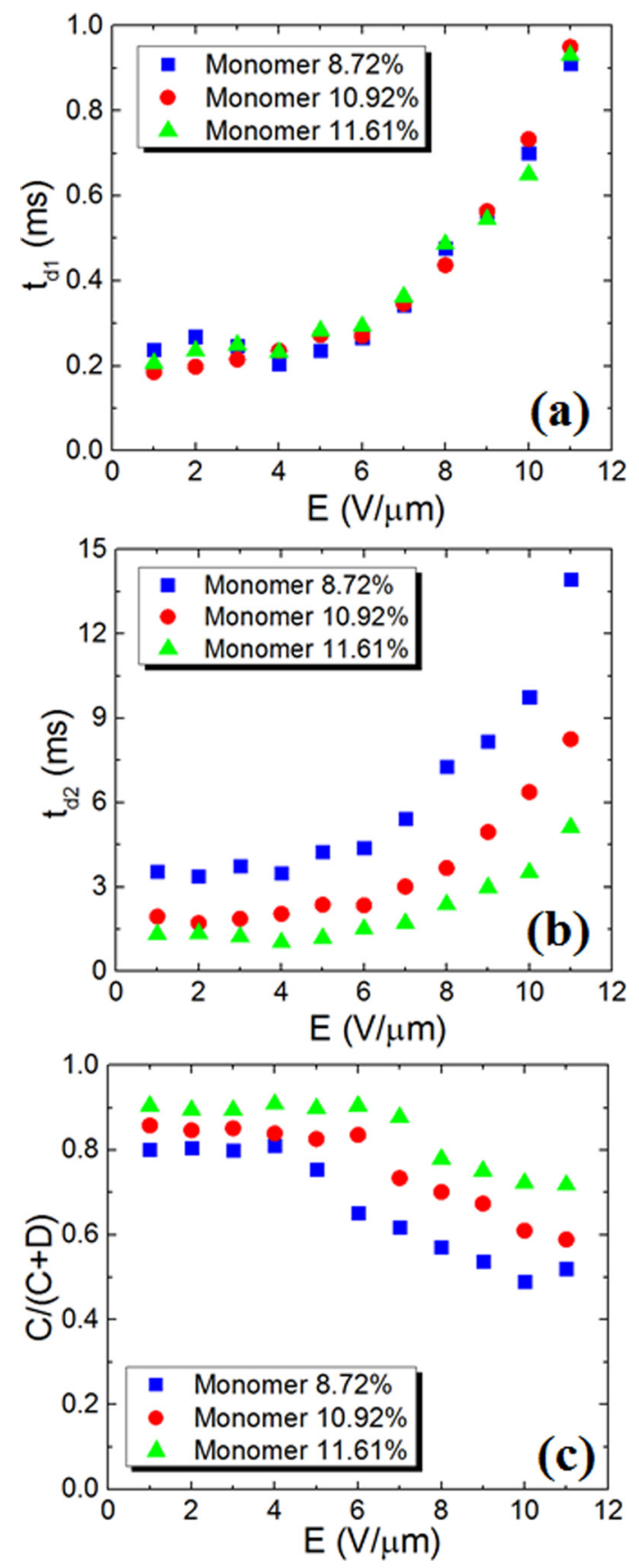

FIG. 5. (a) Kerr effect decay time constants $t_{d 1}$, (b) electrostriction decay time constant $t_{d 2}$, and (c) respective contribution of Kerr effect when different electric fields are applied to samples with different monomer concentrations.

decay time becomes slower when electric field gets stronger. ${ }^{8}$ For a BPLC with higher monomer concentration, its polymer network is stiffer and lattice less distorted, thus, the relaxation time is faster upon removing the electric field. Similar to the aforementioned analysis for rise process, we also plotted $C$ / $(C+D)$ in Fig. 5(c) to compare the respective contribution of Kerr effect and electrostriction to the overall $\Delta n$. It clearly shows that there is also a critical electric field $E_{c}$ for the decay process. When the electric field is weak, i.e., $E<E_{c}$, the local reorientation within double-twisted cylinders dominates. When $E>E_{c}$, the contribution of electrostriction effect gradually increases. In sample 1 , it reaches $\sim 50 \%$ at $E=11 \mathrm{~V} / \mu \mathrm{m}$, as Fig. 5(c) shows. For those samples with 
higher monomer concentrations, their $E_{c}$ is higher and lattice distortion is less so that the electrostriction effect is less. However, the required operation voltage is higher.

The abovementioned double rise/decay model well explains the dynamic response mechanisms of a BPLC composite. From our analysis, both response time and hysteresis of a PS-BPLC increase as the applied voltage exceeds a critical voltage. Therefore, it is essential to keep the operating voltage below the critical voltage in order to achieve fast response time and hysteresis-free performance. This serves as a useful guideline for future BPLC material development and device optimization. On the material side, higher monomer concentration can be used to form more stable polymer network in order to suppress the electrostriction effect, but bear in mind that any excessive polymer would cause the operation voltage to increase undesirably. ${ }^{25,28}$ Therefore, on the material side a delicate balance between response time, hysteresis and operation voltage should be taken into consideration. On the device side, it is strongly desired to keep the operating voltage below the critical voltage of the BPLC composite. For example, the protruded IPS electrode with elliptical shape is useful for reducing electrostriction and suppressing hysteresis. ${ }^{19}$

In conclusion, our proposed model fits well with our experimental results. From the model, we can quantitatively evaluate the contribution of Kerr effect and electrostriction effect of a BPLC at different electric fields. When the electric field is below a critical field, Kerr effect dominates and the response time is usually within submillisecond range and hysteresis is negligible. Once the electric field exceeds the critical field, electrostriction effect gradually manifests. Under such a circumstance, response time gets slower and hysteresis gets larger. Stiffening polymer network helps to suppress electrostriction, but the price paid is increased voltage. To lower operation voltage, a viable approach is to use protruded electrode. Therefore, a low voltage and hysteresisfree BPLC with submillisecond response time can be achieved.

The authors are indebted to Dr. Yasuhiro Haseba of JNC for providing the JC-BP06N sample, Yifan Liu for useful discussion, and Industrial Technology Research Institute (ITRI, Taiwan) for the financial support.

${ }^{1}$ H. Kikuchi, M. Yokota, Y. Hisakado, H. Yang, and T. Kajiyama, Nat. Mater. 1, 64 (2002).

${ }^{2}$ J. Yan, L. Rao, M. Jiao, Y. Li, H. C. Cheng, and S. T. Wu, J. Mater. Chem. 21, 7870 (2011).

${ }^{3}$ Z. Luo, D. Xu, and S. T. Wu, J. Disp. Technol. 10, 526 (2014).

${ }^{4}$ Y. H. Lin, H. S. Chen, H. C. Lin, Y. S. Tsou, H. K. Hsu, and W. Y. Li, Appl. Phys. Lett. 96, 113505 (2010).

${ }^{5}$ J. Yan, Y. Li, and S. T. Wu, Opt. Lett. 36, 1404 (2011).

${ }^{6}$ D. K. Yang and S. T. Wu, Fundamentals of Liquid Crystal Devices (Wiley, New Jersey, 2006).

${ }^{7}$ D. Xu, L. Rao, C. D. Tu, and S. T. Wu, J. Disp. Technol. 9, 67 (2013).

${ }^{8}$ K. M. Chen, S. Gauza, H. Q. Xianyu, and S. T. Wu, J. Disp. Technol. 6, 49 (2010).

${ }^{9}$ Y. Chen, J. Yan, J. Sun, S. T. Wu, X. Liang, S. H. Liu, P. J. Hsieh, K. L. Cheng, and J. W. Shiu, Appl. Phys. Lett. 99, 201105 (2011).

${ }^{10}$ P. Bos, T. Buzak, and R. Vatne, Proc. Soc. Inf. Disp. 26, 157 (1985).

${ }^{11}$ S. Gauza, X. Zhu, W. Piecek, R. Dabrowski, and S. T. Wu, J. Disp. Technol. 3, 250 (2007).

${ }^{12}$ H. C. Cheng, J. Yan, T. Ishinabe, N. Sugiura, C. Y. Liu, T. H. Huang, C. Y. Tsai, C. H. Lin, and S. T. Wu, J. Disp. Technol. 8, 98 (2012).

${ }^{13}$ J. Yan, H. C. Cheng, S. Gauza, Y. Li, M. Jiao, L. Rao, and S. T. Wu, Appl. Phys. Lett. 96, 071105 (2010).

${ }^{14}$ G. Heppke, M. Krumrey, and F. Oestreicher, Mol. Cryst. Liq. Cryst. 99, 99 (1983).

${ }^{15}$ H. Choi, H. Higuchi, and H. Kikuchi, Appl. Phys. Lett. 98, 131905 (2011).

${ }^{16}$ H. S. Kitzerow, P. P. Crooker, S. L. Kwok, J. Xu, and G. Heppke, Phys. Rev. A 42, 3442 (1990).

${ }^{17}$ H. Stegemeyer and E. Porsch, Phys. Rev. A 30, 3369 (1984).

${ }^{18}$ H. S. Kitzerow, Mol. Cryst. Liq. Cryst. 202, 51 (1991).

${ }^{19}$ L. H. Rao, J. Yan, S. T. Wu, Y. C. Lai, Y. H. Chiu, H. Y. Chen, C. C. Liang, C. M. Wu, P. J. Hsieh, S. H. Liu, and K. L. Cheng, J. Disp. Technol. 7, 627 (2011).

${ }^{20}$ Y. Chen, D. Xu, S. T. Wu, S. Yamamoto, and Y. Haseba, Appl. Phys. Lett. 102, 141116 (2013).

${ }^{21}$ D. Xu, Y. Chen, Y. Liu, and S. T. Wu, Opt. Express 21, 24721 (2013).

${ }^{22}$ J. Yan, D. Xu, H. C. Cheng, S. T. Wu, Y. F. Lan, and C. Y. Tsai, Appl. Opt. 52, 8840 (2013).

${ }^{23}$ Y. Liu, Y. F. Lan, H. Zhang, R. Zhu, D. Xu, C. Y. Tsai, J. K. Lu, N. Sugiura, Y. C. Lin, and S. T. Wu, Appl. Phys. Lett. 102, 131102 (2013).

${ }^{24}$ J. Yan, Y. Chen, S. T. Wu, S. H. Liu, K. L. Cheng, and J. W. Shiu, J. Appl. Phys. 111, 063103 (2012).

${ }^{25}$ J. Yan and S. T. Wu, J. Disp. Technol. 7, 490 (2011).

${ }^{26}$ Y. F. Lan, C. Y. Tsai, J. K. Lu, and N. Sugiura, Polymer 54, 1876 (2013).

${ }^{27}$ P. R. Gerber, Mol. Cryst. Liq. Cryst. 116, 197 (1985).

${ }^{28}$ J. Zhu, S. Ni, C. Chen, X. Song, C. Chen, J. Lu, and Y. Su, Liq. Cryst. 41, 891 (2014). 